\title{
Heart Rate Variability (HRV) biofeedback: A new training approach for operator's performance enhancement
}

\author{
Auditya Purwandini Sutarto ${ }^{1}$, Muhammad Nubli Abdul Wahab ${ }^{2}$, Nora Mat Zin ${ }^{3}$ \\ ${ }^{1}$ University of Abmad Dablan (INDONESLA); ${ }^{2}$ Universiti Malaysia Pahang (MALAYSLA); \\ ${ }^{3}$ International Islamic University Malaysia (MALAYSIA) \\ audityaps@yahoo.com; mnubli@,ump.edu.my;noramz@,iiu.edu.my
}

Received October 2009

Accepted May 2010

\begin{abstract}
The widespread implementation of advanced and complex systems requires predominantly operators' cognitive functions and less importance of human manual control. On the other hand, most operators perform their cognitive functions below their peak cognitive capacity level due to fatigue, stress, and boredom. Thus, there is a need to improve their cognitive functions during work. The goal of this paper is to present a psychophysiology training approach derived from cardiovascular response named heart rate variability (HRV) biofeedback. Description of resonant frequency biofeedback - a specific HRV training protocol - is discussed as well as its supported researches for the performance enhancement. HRV biofeedback training works by teaching people to recognize their involuntary HRV and to control patterns of this physiological response. The training is directed to increase HRV amplitude that promotes autonomic nervous system balance. This balance is associated with improved physiological functioning as well as psychological benefits. Most individuals can learn HRV biofeedback training easily which involves slowing the breathing rate (around six breaths/min) to each individual's resonant frequency at which the amplitude of HRV is maximized. Maximal control over HRV can be obtained in most people after approximately four sessions of training. Recent studies have demonstrated the effectiveness of HRV biofeedback to the improvement of some cognitive functions in both simulated and real industrial operators.
\end{abstract}


Keywords: heart rate variability, biofeedback, operator, cognitive performance, resonant frequency

\section{Introduction}

To address with global challenge, a great number of environments have been upgrading into the higher-tech, higher value-added and more sophisticated work system. The increase of such advanced and complex systems shifts the nature of the human operator's task from an emphasis on perceptual motor skills or psychomotor activities to an emphasis on knowledge-based or cognitive activities. On the other hand, most operators work below their peak cognitive level. There are a number of block factors that can adversely affect cognitive performance, including fatigue, boredom, and stress (Megaw, 1979). Reduced cognitive performance may continue to poor outcomes such as more error, low productivity which in turn cause financial loss.

Pattyn, Neyt, Henderickx, and Soetens (2008) showed that the crucial determinant of cognitive performance is the ability to maintain sustained attention or vigilance on a given stimulation source or task. The vigilance decrement has been described as a slowing in reaction times or an increase in error rates. Radar operators can have a $70 \%$ drop in efficiency within 30 min of commencing a trial, through boredom (Fox, 1971). The result of Clock Test from Mackworth's study also showed a $12 \%$ decline in the frequency of signal detections after only 30 minutes of performing the task and an $18 \%$ drop by the end of the 2 -hour session (Gluckman, 1990). In addition to attention and vigilance, short-term memories (STM) also can be very unstable. Most people have difficulty remembering even three items after 18 seconds and Marsh, Hicks, and Landau (1997) presented that STM can decay within 2 seconds.

Researches have studied many strategies to deal with the maintenance of vigilance and attention in conditions of monotonous or versatile activity. One of these strategies is by providing background music during work. According to a study completed by Hallam, Price, and Katsarou (2002), calming music led to better participant performance on an arithmetic task and a memory task than no music. It 
was also found that background music on cognitive test performance led to improved performance when compared with a control condition (Cockerton, Moore, \& Norman, 1997). However it may also negatively affect human performance in such tasks as attention and vigilance (Dalton \& Behm, 2007). In addition to providing music, variety kinds of training such as cognitive training (Willis et al., 2006), motor-imagery training (Papadelis, Papadeli, Bamidis, \& Albani, 2007), and knowledge-based training (Gramopadhye \& Wilson, 1997) have been suggested as powerful tools for enhancing cognitive functions through modifying individuals' skills or attitudes mechanism. Yet, a great number of studies on cognitive training are aimed to cope with cognitive aging rather than improve cognitive functions among operators or workers. Moreover, Papadelis et al., (2007) have examined the effectiveness of mental imagery training on cognitive performance. The results revealed significantly higher performance level on the imagery-training group than the control group. However, this study emphasized more on psychomotor performance in a task where spatiotemporal or dynamic control of the action is highly required. For simple tasks in real work settings, cognitive and psychomotor functions overlap to the extent that the stimuli require thought, but if the stimuli are complex and require decisions about how to respond, and then the task becomes more cognitive (Wetherell, 1997). Imagery training hence might be less appropriate to be employed in a wider range of tasks particularly those assigned in more cognitive-demanding work systems. Nevertheless, to date, no psychophysiology approaches have been used to improve operators' cognitive performance. Through applied psychophysiology strategy along with various senses stimulation and with the operators' active involvement, operators are trained how to self-regulate their states. In this paper we present the basic concept of psychophysiological approach and its potential application in performanceenhancement. Description of resonant frequency biofeedback, a specific training protocol, is also discussed as well as its supported researches for the improvement of cognitive performance.

\section{Psychophysiology and biofeedback}

The success of operator's performance in work systems depends not only on their health status, general, and professional training, but also on their specific ability to maintain the required cognitive functional level for a long period of work time. To ensure such conditions, the development of a safe method to deal with 
performance block factors is needed. Psychophysiological self-regulation is a training approach that can be employed to achieve optimal performance through biofeedback "learning" method.

Psychophysiological self-regulation refers to the ability of a person to control affective and cognitive states based on autonomic and central nervous system functioning (Prinzel, Pope, \& Freeman, 2001). The technique utilizes physiological indicators of these states and provides feedback so that the person learns these associations and how to modulate their occurrence in order to achieve the optimal human physiological functions. The correction of these functions is supported by the growing of computer systems with biofeedback in various modalities (bioengineering systems). Visual, auditory, or tactile stimulation are used and, with the subjects' active involvement, self-regulation and self control skills can be acquired and used to correct of their states toward the optimal (Schwartz \& Schwartz, 2003). Psychophysiological self-regulation is also commonly termed as biofeedback (biological feedback) training.

Over the past 50 years, researchers have used biofeedback to do everything from helping people relax to treating severe headaches, chronic pain, and high blood pressure. Currently, biofeedback has been applied in wider areas, not only for clinical purposes but also for the enhancement of sport and work performance. Following are several machines and techniques of biofeedback commonly used (Schwartz \& Schwartz, 2003; Sherman, 2004).

- Surface Electromyogram (sEMG) biofeedback. A typical placement of sEMG sensor is the frontalis (forehead) or the trapezius (shoulder) muscles. As muscles become tenser, they make more electricity. SEMG biofeedback is mainly used to promote the relaxation of those muscles involved in backaches, headaches, neck pain and grinding one's teeth. The feedback from sEMG display helps clients learn to relax overly tense muscles, better activate weak muscles, or change the coordination pattern among agonist, antagonist, and synergist muscles

- Temperature biofeedback. Thermistors are used to record skin temperature (usually from the fingertip) in order to track near-surface blood flow. Nearsurface blood flow changes with sympathetic activity, pain, and many 
pathological conditions. The goal of the training is directed to control client's temperature.

- Electroencephalogram (EEG) biofeedback or neurofeedback. Neurofeedback is a sophisticated form of biofeedback based on specific aspects of cortical activity. The brain's electrical activity is recorded from sensors mounted on the surface over various parts of the brain. The goal of neurofeedback training is to teach the individual what specific states of cortical arousal feel like and how to activate such states voluntarily

- Heart Rate Variability (HRV) biofeedback. HRV represents the beat to beat changes in the interbeat interval (time between two successive R-waves). HRV biofeedback training is aimed to teach people change tonic level of physiological arousal by increasing HRV amplitude (Lehrer, 2007). The monitoring of HRV activity is detected through electrocardiogram (ECG) or photoplethysmography (PPG). Respiration measure is usually incorporated into HRV biofeedback. This dual method is particularly useful in learning how to breath abdominally (diaphragmatically), and therefore in any conditions to do with the respiratory system.

Currently very few works have been done on the use of biofeedback training (note: psychophysiological/biofeedback training can be used synonymously) for performance enhancement in healthy subjects, particularly for improving cognitive functions. One of the few studies that have been undertaken was reported by Prinzel et al. (2001) on the use of neurofeedback in conjunction with adaptive automation to minimize the onset of hazardous state of awareness. They found that psychophysiological self-regulation could enhance cognitive resource management skills of operators. The participants who had received neurofeedback training performed significantly better in controlling hazardous states of awareness and reported lower subjective mental workload than participants in the control group. Egner and Gruzelier, (2003, 2004) and Vernon (2003) have conducted other researches on neurofeedback in healthy subjects. These studies result in improved attention and decreased impulsiveness that were indicated by less error of both omission and commission, and shorter reaction times. Furthermore, Vernon (2005) reviewed evidence from neurofeedback training studies within three main areas; sport, cognitive, and artistic performance. He concluded that it seemed reasonable 
to utilize neurofeedback as a tool to train individuals to re-create patterns of cortical activity in an attempt to enhance performance. Nevertheless, despite its potential applicability, neurofeedback has drawbacks to its high cost and less practical for wide range of applications mainly in work place settings.

In this paper, we present HRV-biofeedback as a potential tool for cognitive performance enhancement. HRV biofeedback is designed to control oscillatory variability in heart rate, hence directly targeting and exercising the body's own physiological control mechanisms. Most other biofeedback methods influence these mechanisms more indirectly by teaching people to control tonic level of various physiological functions (e.g., muscle tension, finger temperature, heart rate, blood pressure, etc). In fact, controlling tonic levels is noticeably more difficult than learning to increase HRV (Lehrer, 2007). Moreover, compared to neurofeedback, HRV feedback is much simpler and more straightforward to learn and use, which facilitates rapid improvement. Its cost-effectiveness also makes it accessible to a greater number of people and in variety domains (McCraty \& Tomasino, 2004).

The mechanism of HRV-biofeedback is illustrated in figure 1. Two types of sensors, pulse wave (heart rate) and respiration sensor detect each pulse and respiration pattern respectively. The time interval between consecutive heartbeats and breaths per minute are computed. The changing beat-to-beat heart rate and breathing pattern are then displayed on the computer screen. HRV-biofeedback training works by teaching people to recognize their involuntary HRV and to control patterns of this physiological response following the reference signals. There are several HRV training strategies which can effectively be used to increase cardiac variability in a health enhancing way including psychophysiological or heart rhythm coherence feedback, (McCraty 2003), or oscillatory biofeedback (Suvorov, 2006), and resonant frequency training (Lehrer, Vaschillo, \& Vaschillo, 2000). 


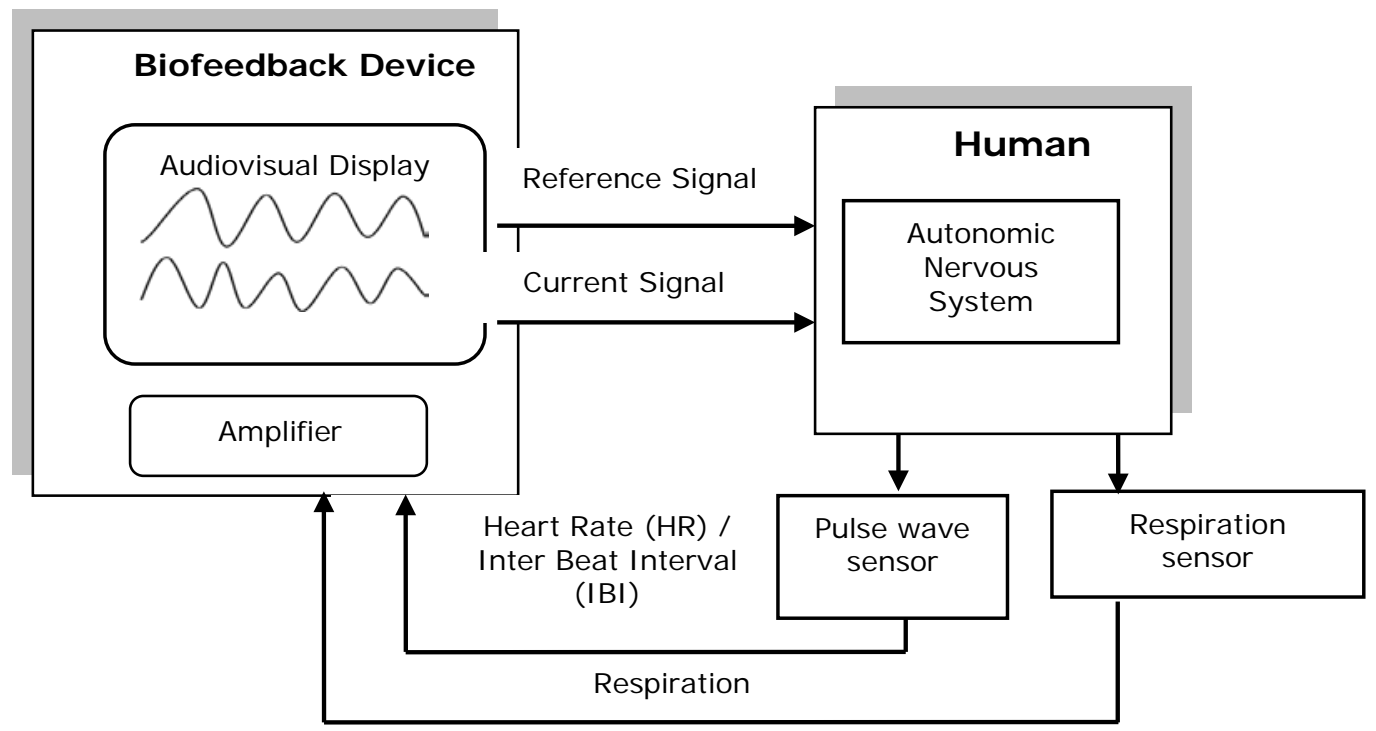

Figure 1. "Scheme of HRV- Biofeedback". Source: Own contribution.

The Heart Math Institute has conducted extensive researches on the use psychophysiological coherence. The coherent mode is represented by a sine wavelike pattern in the heart rhythms and a narrow-band, high-amplitude peak in the low frequency range of the HRV power spectrum, at a frequency of about 0.1 hertz. At the physiological level, this mode is characterized by increased efficiency and harmony in the activity and interactions of the body's systems. While psychologically, this mode is correlated with reduced perceptions of stress, increased emotional balance, and enhanced cognitive performance (BarriosChoplin, McCraty, \& Cryer, 1997; McCraty, 2002).

In an exploratory field study, Barrios-Choplin et al. (1997) found that an Inner Quality Management (IQM) training on a group of Motorola employees (30 factory workers) significantly increased productivity through the improvement of job satisfaction and communication, and through the reduction of tension and anxiety. HRV biofeedback strategy was one of four modules intervened in the 3-month study. McCraty (2002) also evaluated the efficacy of this technique on cognitive performance in an auditory discrimination task. In this investigation, 30 subjects were randomly divided into matched control and experimental groups based on age and gender. Cognitive performance was assessed by determining subjects' reaction times in an oddball auditory discrimination task before and after practicing the HRV-biofeedback technique to increase cardiac coherence. As compared to the 
control group, the experimental group subjects using HRV-biofeedback technique demonstrated a significant increase in heart rhythm coherence and a significant decrease in reaction times $(p<0.05)$ in the discrimination task following the application of the technique. These findings support the hypothesis that the changes in brain activity that occur during states of increased psychophysiological coherence direct to changes in the brain's information processing capabilities.

In addition to psychophysiological coherence, Suvorov (2006) proposed that the presence of inherent harmonics or respiratory sinus arrhythmia (RSA) is one of the qualitative criteria for the efficiency improvement of operator activity. RSA refers to cyclical oscillation in heart rate coincident with the respiratory cycle, such that increases in heart rate occur during inhalation and decreases in heart rate during exhalation (Berntson et al., 1997). The result of this study showed that 14 across 15 subjects without inherent harmonics developed harmonics after a series of 8 12 sessions of cardiorhythm biofeedback control. Their performance also improved significantly, making fewer errors $(p<0.01)$ and increasing the rate of information processing on psychomotor activity $(p<0.01)$ at post-training testing. This biofeedback control directed to strength, restore or create harmonics associated with respiratory movements by doing specific breathing rhythm. The presence of inherent harmonics remains stable for a prolonged period of time which has been confirmed in a single follow-up study of cardiac rhythm after $1 \frac{1 / 2}{2}$ years. The results from those previous studies are encouraging and indicate the possible effectiveness of the use of HRV-biofeedback in work place settings, particularly for improving operators' cognitive performance.

\section{Resonant Frequency Training (RFT) biofeedback}

Over other training criteria just described (McCraty, 2003; Suvorov, 2006), resonant breathing appears to become the most promising strategy being applied at workplace. It is a well-established technique for more than 25 years of research endeavors (Vaschillo, Lehrer, Rishe, \& Konstantinov, 2002). All of HRV biofeedback strategies are essentially directed to augment the amplitude of HRV. However over other strategies, Resonant Frequency Training (RFT) biofeedback demonstrates how to obtain the high amplitude instead of simply produce a smooth or sine wavelike pattern of heart rhythm. Compared to psychophysiological coherence, RFT technique works by allowing subjects to gain control of their physiology rather than 
relax under pressure. A study of RFT in 54 healthy subjects, assigned randomly 23 to the biofeedback group and 31 to the waiting list group found that none of the physiological changes in biofeedback group were closely associated with selfreported experiences of relaxation (Lehrer et al., 2003). This result suggested that the cardiorespiratory effects cannot be explained by relaxation whereas other HRV biofeedback techniques use the relaxation as the mediating role in enhancing performance. In real work settings, cognitive performance decrement is influenced by either stress or fatigue (Pattyn et al., 2008) rather than anxiety. Therefore, RFT presents opportunities to be applied in a larger range of tasks. Moreover, regarding with number of training sessions, RF biofeedback training requires only four sessions (Lehrer, 2007), irrespective of age, to teach the subjects acquire the targeted resonant breathing while oscillatory biofeedback may require up to 15-20 sessions to get inherent harmonics (Suvorov, 2006).

Resonant frequency training is a specific biofeedback training strategy that is aimed at producing maximal increases in amplitude of respiratory sinus arrhythmia (RSA) (Lehrer et al., 2000). RSA is a component of HRV which reflects homeostatic activity and adaptability (Berntson et al., 1997). It is now recognized that the variability (rhythm) of a heart is an indicator of both physiological resiliency and behavioral flexibility, reflecting the person's capacity to adapt to stress and environmental demands (Andreassi, 1995). A high degree of instability or too little variation can be detrimental to efficient physiological functioning. Recent research has showed that diminished HRV is a sign of vulnerability to stress, whether it be from psychological or physical stress or from the diseases (Lehrer, 2007), thus optimum variation is essential.

The RFT biofeedback procedure developed by Lehrer et al. (2000) is based on resonance properties of the cardiovascular system. They suggest that RFT can mediate a homeostatic state in the body. To generate high-amplitude oscillation in autonomic functions, the procedure uses slow-paced breathing at each individual's resonant frequency, at about $0.1 \mathrm{~Hz}$ ( 6 breaths/minute). The $0.1 \mathrm{~Hz}$ is in the lower frequency band which reflects the sympathetic and parasympathetic autonomic control and reflects the action of the baroreflex. The baroreflexes are important mechanisms for control of blood pressure (Eckberg \& Sleight, 1992). Vaschillo, Vaschillo, and Lehrer (2006) have shown that HRV and respiration are exactly in phase with each other when individual breathe at this specific frequency (i.e. heart 
rate rises simultaneously with inhalation and decreases simultaneously with exhalation). HRV biofeedback thus should maximize respiratory efficiency.

Most individuals can learn RFT biofeedback training easily which is combined with instructions to breathe diaphragmatically, to inhale through the nose, and to exhale through pursed lips, as well as do prolonged exhalation. Maximal control over HRV at the resonant frequency is achieved in most people after approximately four sessions of training (Lehrer, 2007). As RSA increases, simultaneously the spectral distribution of HRV shifts, with a greater percentage of total variability now existing in low frequency (LF) range, includes the $0.1-\mathrm{Hz}$ point. Increase in LF HRV during biofeedback practice reflects resonance effects involving both RSA and baroreflex gain (Lehrer et al., 2003).

Even though very few works have particularly been done with operators (Sutarto \& Abdul Wahab, 2008; Sutarto, Abdul Wahab, \& Mat Zin, 2009), applying resonant frequency biofeedback shows promise in clinical and non-clinical populations and may be generalized to work settings. Lehrer et al. (2000) have used breathing techniques guided by heart rate biofeedback in laboratory settings in healthy subjects. They found that when breathing is combined with HRV biofeedback, subject in training group were able to create resonance in the cardio respiratory system between the effects of respiration and those of the baroreflex. Consistent, daily practice of this method also increases total heart rate variability with almost all of the oscillations peaking at a single frequency $(0.1 \mathrm{~Hz})$. Furthermore, Lehrer et al. (2003) also demonstrated the neuroplasticity effect of the baroreflex that suggests the increase of baroreflex may affect and improve autonomic regulation throughout the day among individuals trained in this method.

\section{Procedure of resonant frequency training}

Lehrer et al. (2000), has published a manual procedure for HRV biofeedback training. The participants receive 5 - 6 sessions, each 30 - 50 minutes, one session per week. The main concept of the training is to assist operators to determine their resonant frequency at which maximum amplitudes of HRV are generated and to teach operators to breathe according to their specific frequency. A combination of slow abdominal breathing and a positive emotion maximizes HRV in the LF spectrum because it superimposes the effects of three oscillators: 
breathing, autonomic activity blood, and pressure regulation (Moss, 2004). As the training continues, they can voluntarily maintain the changes in their heart rhythm patterns, which generally become more regular, more sine-wave like and produce greater amplitude.

At the first session, the trainee is first taught to breathe at their resonant frequency i.e., the frequency at which maximum amplitudes of HRV are generated voluntarily for each individual. The resonant frequency is determined in the first session by measuring HR oscillation amplitudes while the individual breathed for intervals of 2-3 minutes at each of the following frequencies: $6.5,6.0,5.5,5.0$, and 4.5 breaths/min. A pacing stimulus: a light display that moved up and down on the computer screen will guide the participants to breathe at the target respiratory rate and when to inhale and exhale. The resonant frequency is determined as the respiratory frequency producing the highest power peak, at the respiratory frequency, on a moving Fourier analysis of HR, which was displayed with reference to the past $30 \mathrm{~s}$, updated every second. At the end of first session, the trainee is instructed to practice breathing at his/her resonant frequency on a daily basis, minimum of 5 -minutes (20 minutes/day total) practice segments. Throughout training, the individual is encouraged to breathe shallowly and naturally, in order to avoid hyperventilation, as can be provoked by this technique (Lehrer, Carr, \& Smetankine, 1997).

At the second session, the trainees are asked about their experiences with resonant-frequency breathing within the past week. In this session, they are trained to do relaxed abdominal breathing. They are also taught to inhale through the nose and exhale through pursed-lips with exhalation longer than inhalation.

In the third session, participants are given biofeedback and step by step taught to maximize the peak amplitude of RSA. This aspect of training is repeated until subjects are able to meet the criteria of maximizing spectral activity near $0.1 \mathrm{~Hz}$ while maintaining diaphragmatic breaths. As a manipulation check for the biofeedback, the percentage of LF activity and baroreceptor power are monitored during training sessions.

In all subsequent sessions, the primary task is to monitor progress, measure the HRV parameters, and correct errors in technique. An incorporation of a combination of mental and physical stressor (e.g. noise) during these sessions is suggested. 
Such stressors help the trainee gain proficiency in controlling performance pressure and autonomic activity in real work setting. Common mental stressor used in biofeedback training is arithmetic test which may take form of " $x+y-z=$ ". (Sharpley, 2000) or "serial sevens" (subtract by sevens from a given number) (Strack, 2003).

\section{Studies on the use of RFT biofeedback for performance enhancement}

Strack (2003) used resonant biofeedback strategy for improving batting performance in baseball. Forty three baseball players were randomly assigned into training $(n=22)$ and control group $(n=21)$. Participants performed a competitive batting contest and a six week RFT biofeedback. Participants were measured on batting performance and self-report of state anxiety (CSAI-2), flow (FSS-2), and a visual analog scale (VAS) of how well the baseball was tracked visually. Results showed that biofeedback group showed a $60 \%$ improvement compared to a $21 \%$ improvement for the control group. Partial support was found on the occurrence of the subjective state of flow while prediction made for the VAS and subscales on the CSAI-2 were not confirmed. Furthermore, more recent studies have demonstrated that RFT could improve several cognitive functions in both university students acted as simulated operators (Sutarto \& Abdul Wahab, 2008) and real operators (Sutarto et al., 2009). The method and results of both studies will be described in the following subsections.

\subsection{Method}

In a preliminary study, nine female university students who acted as simulated operators received 6 sessions of HRV biofeedback during 3 weeks, two sessions per week. The effect of biofeedback training were examined by administering three cognitive task: Stroop Color-Word Test (cognitive flexibility), memory (verbal memory), and arithmetic test (decision making). As a measure of training progress, the percent of total HRV shifted to the LF range was assessed between sessions using Biofeedback Stress Management Kit developed by Institute of Molecular biology and Biophysics, Russian Academy of Medical Sciences.

A replication of this study using control design has been also conducted with real operators as experimental subjects (Sutarto et al., 2009). Twenty female manufacturing operators participated in the study and were randomly assigned to 
the intervention and control group. One participant in the training group was unable to register a finger pulse and was excluded, 3 subjects in control group dropped out before completion. The final sample used in analyses was 16 participants, 9 in training group and 7 in control group, who completed both pre to post- training assessment.

In the first meeting after each participant read and signed an informed consent, a 10-min physiological stress profile assessment was administered individually. The percentage of LF power of HRV spectral activity was recorded during 4-minute baseline, 2-minute stressor ("serial seven"), and 4-minute recovery period. After that, participants in the both groups performed cognitive tests (Stroop test, Sternberg test, and D2 attention test). The post assessment was carried out one week after the final training period, thus allowing for some measure of maintenance.

Training participants received five session of weekly HRV biofeedback training of 30-50 minutes each. In the session 4 and 5 , subjects in the biofeedback group also performed mental arithmetic (stressor) task with the goal of helping subjects gained proficiency in controlling performance pressure and autonomic activity. The control group attended five sessions and was monitored physiologically without receiving special instruction. Physiological measurements were collected by means of biofeedback system using an I-330 C-2 interface (J \& J Engineering, Poulsbo, WA).

\subsection{Results}

Overall, there was an increase in cognitive performance after training both in simulated and real operators. Paired t-test was used to evaluate whether any improvement on several cognitive functions among university students after training. The memory and decision making showed statistically improved from pre to post intervention ( $z=-2.536, p<0.05$, and $z=-2.12, p<0.05$, respectively). However, their cognitive flexibility measured by Stroop test showed no significant improvement $(z=-1.599, p>0.05)$. Further analysis revealed a small effect of stroop test $(r=0.24)$ while a medium $(r=0.47)$ and large effect $(r=0.71)$ for arithmetic test and memory test, respectively. 
Due to the violation of the sphericity assumption, training effectiveness between sessions was assessed using Friedman's ANOVA test with repeated measures on percent of total HRV power shifted to the LF range. Result revealed a significant change toward LF range over all training sessions $\left(\chi^{2}=34.044, p<0.001\right)$. As post hoc test, Wilcoxon signed-rank tests were used to follow up this finding. A Bonferroni correction was applied and so all effects are reported at 0.0125 level of significance ( 0.05 divided by four comparisons). The result show that the LF oscillation significantly increase from the first week of the training to the second week, $Z_{12}=-2.429, r_{12}=-0.572$, and the following subsequent weeks, as summarized in table 1.

\begin{tabular}{|c|c|c|c|c|}
\hline & $\begin{array}{c}\text { Session 1 - } \\
\text { Session 2 }\end{array}$ & $\begin{array}{c}\text { Session 2 - } \\
\text { Session 3 }\end{array}$ & $\begin{array}{c}\text { Session 4 - } \\
\text { Session 3 }\end{array}$ & $\begin{array}{c}\text { Session 5 - } \\
\text { Session 4 }\end{array}$ \\
\hline $\mathbf{Z}$ & $-2.429^{\mathrm{a}}$ & -2.666 & -2.310 & -2.666 \\
\hline $\mathbf{p}$ & $0.006^{*}$ & 0.002 & 0.010 & 0.002 \\
\hline Size effect & -0.572 & -0.628 & -0.544 & -0.628 \\
\hline
\end{tabular}

a Based on negative ranks

* 0.0125 level of significance

Table 1. "Pairwise comparison of LF Oscillation Change between Subsequent Sessions in Simulated Operators". Source: Own contribution.

In the replication study, repeated measures analyses of variance (RM ANOVAs) was conducted to test the differential efficacy of the training for improving cognitive performance. There was significant group $x$ time interactions on either Sternberg or D2 Attention test but no significant interaction effect was present on Stroop test. Within-group analyses using paired t-tests showed that the intervention group significantly improved cognitive performance from pre-intervention to postintervention on Stroop test (interference score, $\mathrm{t}(8)=-3.442, \mathrm{p}<0.01$ ), Sternberg test (response times, $\mathrm{t}(8)=3.175, \mathrm{p}<0.05$ ), and $\mathrm{D} 2$ attention test (concentration performance, $\mathrm{t}(8)=-3.690, \mathrm{p}<0.01$ ). Partial support was found for the reduction of the DASS score at post intervention. The control participants showed no improvements on any cognitive functions. The performance improvement was also supported with a significant increased in the percent of total low frequency (LF) power in the heart rate spectrum across sessions $\left(\chi^{2}=14,578\right.$, $\mathrm{p}<0.01)$ as summarized in table 2. 


\begin{tabular}{|c|c|c|c|c|}
\hline & $\begin{array}{c}\text { Session 1 - } \\
\text { Session 2 }\end{array}$ & $\begin{array}{c}\text { Session 2 - } \\
\text { Session 3 }\end{array}$ & $\begin{array}{c}\text { Session 4 - } \\
\text { Session 3 }\end{array}$ & $\begin{array}{c}\text { Session 5 - } \\
\text { Session 4 }\end{array}$ \\
\hline $\mathbf{Z}$ & $-0.889^{\mathrm{a}}$ & -2.547 & -0.415 & -2.431 \\
\hline $\mathbf{p}$ & $0.213^{*}$ & 0.004 & 0.367 & 0.006 \\
\hline Size effect & -0.209 & -0.600 & -0.098 & -0.573 \\
\hline
\end{tabular}

a Based on negative ranks

* 0.0125 level of significance

Table 2. "Pairwise comparison of LF oscillation change between subsequent sessions in real Operators". Source: Own contribution.

Figure 2 provides illustration of session improvement in LF activity across five sessions in both groups. Although Wilcoxon test showed that a significant increase of LF shift was not found in all consecutive sessions among real operators (Sutarto et al., 2009), but in general both groups learned the technique effectively from first to last session. Similar findings were also found for cognitive performance as shown in table 3.

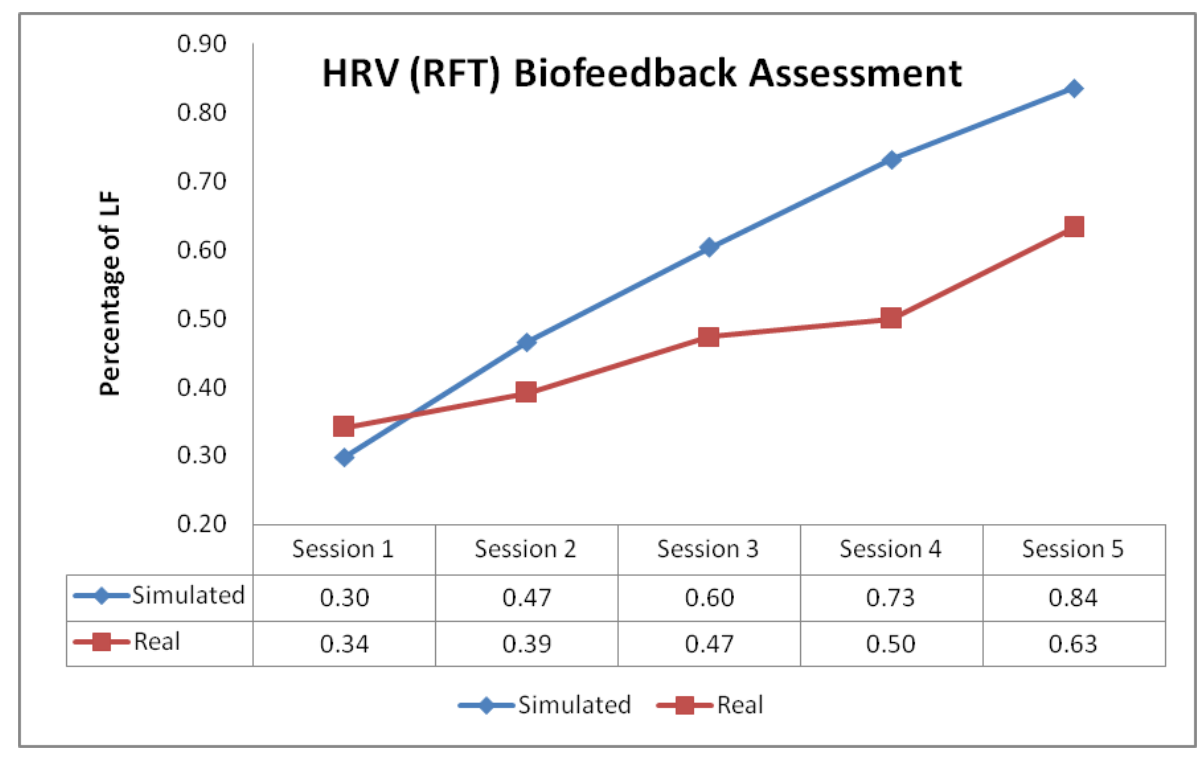

Figure 2. "Change in percentage of Low Frequency (LF) activity throughout five Sessions in simulated and real operators". Source: Own contribution. 
Simulated Operators

\begin{tabular}{|c|c|c|c|c|}
\hline 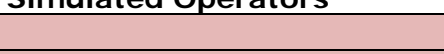 & \multicolumn{4}{|c|}{ Mean (Standard Deviation) } \\
\hline Cognitive Measure (Score) & Pre & Post & $\mathbf{p}$ & $\begin{array}{c}\text { Size } \\
\text { Effect } \\
\left(r^{2}\right)\end{array}$ \\
\hline $\begin{array}{l}\text { Cognitive Flexibility } \\
\text { (interference score) }\end{array}$ & $53.5(8.06)$ & $58.4(8.57)$ & 0.076 & 0.24 \\
\hline $\begin{array}{l}\text { Verbal Memory (correct } \\
\text { answer) }\end{array}$ & $7(3.3)$ & $10(2.8)$ & 0.010 & 0.71 \\
\hline $\begin{array}{l}\text { Decision Making (correct } \\
\text { answer) }\end{array}$ & $2(1.4)$ & $4(2.2)$ & 0.014 & 0.47 \\
\hline \multicolumn{5}{|l|}{ Real Operators } \\
\hline $\begin{array}{l}\text { Cognitive Flexibility } \\
\text { (interference score) }\end{array}$ & $65.36(6.64)$ & $70.25(4.31)$ & 0.005 & 0.60 \\
\hline $\begin{array}{l}\text { Short term Memory (response } \\
\text { times in ms) }\end{array}$ & $\begin{array}{l}1853.47 \\
(503.64) \\
\end{array}$ & $\begin{array}{l}1397.09 \\
(164.70)\end{array}$ & 0.007 & 0.56 \\
\hline $\begin{array}{l}\text { Attention (concentration } \\
\text { performance) }\end{array}$ & $103.8(34.68)$ & $150.4(36.70)$ & 0.003 & 0.63 \\
\hline
\end{tabular}

Table 3. "Summary of cognitive performance assessment in simulated and real operators". Source: Own contribution.

\section{Summary and conclusion}

A novel psychophysiological approach is proposed to solve problems related with human operator's cognitive performance. The resonant frequency training (RFT), a specific of heart rate variability (HRV) biofeedback strategy showed the potential to be an inexpensive psychophysiological approach to improve autonomic balance and respiratory control. An optimal autonomic homeostasis and respiration function is believed to be a mediator for optimum cognitive functioning. This technique combines paced breathing at about $0.1 \mathrm{~Hz}$ ( 6 breaths per minute) along with abdominal and pursed-lips breathing to achieve maximal increases in amplitude of HRV. Most operators can learn easily and rapidly the RFT and afterwards employ it to improve their cognitive functions during work.

The results of studies by Sutarto and Abdul Wahab (2008) and Sutarto et al. (2009) indicated that subjects received biofeedback training improved their cognitive performance significantly. These findings were coupled with a highly significant shift in total power on the heart rate spectral analysis toward the LF range over all sessions. LF is usually under control of both the sympathetic and parasympathetic nervous systems and correlates highly with baroreflex gain (Berntson et al., 1997). It includes the $0.10 \mathrm{~Hz}$ (resonant) component. This verifies that the participants learned how to effectively manipulate and control activity in the autonomic nervous system which continued to autonomic balance. Furthermore, an increase of percentage of LF activity found was in line with performance improvement. It shows 
that being in a state of "resonance" or balanced ANS was linked to enhanced performance. Thus, this study add to the body research that resonant breathing biofeedback training with an increase in HRV indices and an improvement of cognitive functions in healthy subjects.

Lehrer et al. (2000) have developed a theoretical explanation of the mechanism involved in creating resonance in the body. It appears that the slow paced breathing technique learned through HRV-biofeedback creates a resonance between the cardiovascular and the respiratory systems. It is proposed that the physiological mechanism explaining resonance and autonomic balance involve a complex interplay between heart rate and vascular tone baroreflex loops

McCraty and Tomasino (2004) and McCraty (2002) proposed the existence of harmonious interactions of the body's subsystem termed "physiological coherence". They summarized the research that supports the concept that as cardiac afferent neurological input to the brain increases, homeostatic regulation and cognitive processing also increases. They suggest that a combination of rhythmic breathing and the intentional self-induction of a sincere positive emotional state facilitate coherence in the autonomic nervous system. When heart-brain dynamics are modified in this way, the brain's information processing capabilities may change. The changes direct to potential improvement in abilities such as focused attention, discrimination, and motor skills. Such skills are crucial to the work settings and are often under-trained for operators (McCraty, 2003).

Regarding its practical issue, there are several things should be considered before applying HRV biofeedback training. First it is important to consider the trainer's skill. In addition to biofeedback knowledge, a good trainer should have coaching and managerial skills. Lack of personal attention from the trainer may result in incomplete understanding of the techniques and decreased motivation to practice (Kelley, 1995). Second, concerning with lack of time to practice outside sessions, providing the participants with more tips to practice at workplace is recommended. Third, another important aspect of workplace interventions is that of continued training. Workers may be motivated to practice the technique throughout the training and after completing the training, but adherence tends to decrease with time. Offering "refreshment" session three to six months after the completion of the training can provide workers not only with increased motivation to adhere to the 
practice of the technique but also with reminders on the correct method of practicing the technique. Moreover, to be effective in organizational level's goal, HRV biofeedback training should be combined with other strategies or intervention. It is expected that multi-modal performance enhancement techniques may lead to greater outcomes and long-term benefits.

Nevertheless, there were several limitations to the studies. Both studies (Sutarto \& Abdul Wahab, 2008; Sutarto et al., 2009) had small sample sizes, all women, which limited generalizability of our results. Studying operators in other industries and among men may allow us to explore various characteristics that may affect the results found in these studies. Definitive conclusion also cannot be made about the role of autonomic changes in improving cognitive functions. Future studies may further clarify the interrelationship between autonomic nervous sytem and cognitive functioning and its associated features. Furthermore, it is still unclear whether the immediate training effects enlarge, decrease, or remain consistent over time. Longitudinal research is thus warranted. In spite of the shortcomings, the findings of these studies (Sutarto \& Abdul Wahab, 2008; Sutarto et al., 2009) demonstrate the potential application of HRV biofeedback as a useful strategy for enhancing cognitive functions among human operators.

\section{References}

Andreassi, J. L. (1995). Psychophysiology. Hillsdale, New Jersey, USA: Lawrence Erlbaum \& Association.

Barrios-Choplin, B., McCraty, R., \& Cryer, B. (1997). An inner quality approach to reducing stress and improving physical and emotional well-being at work. Stress Medicine, 13, 193-201.

doi: 10.1002/(SICl) 1099-1700(199707)13: 3<193: : AID-SMI 744>3.0.CO;2-I

Berntson, G. G., Bigger, J. T., Eckberg, D. L., Grossman, P., Kaufmann, P. G., Malik, M., Nagaraja, H. N., Porges, S. W., Saul, J. P., Stone, P. H., \& Van Der Molen, M. W. (1997). Heart Rate Variability: origins, methods, and interpretive cavats. Psychophysiology, 34, 623-648.

doi: 10.1111/j.1469-8986.1997.tb02140.x 
Cockerton, T., Moore, S., \& Norman, D. (1997). Cognitive test performance and background music. Perceptual and Motor Skills, 85, 1435-1438.

Dalton, B. H, \& Behm, D. G. (2007). Effects of noise and music on human and task performance: A systematic review. Occupational Ergonomics, 7 (3), 143 - 152.

Eckberg, D. L., \& Sleight, P. (1992). Human baroreflexes in health and diseases. Oxford: Clarendon Press.

Egner, T., \& Gruzelier, J. H. (2003). Ecological validity of neurofeedback: modulation of slow-wave EEG enhances musical performance. NeuroReport, 14, 225- 1228.

Egner, T., \& Gruzelier, J. H. (2004). EEG biofeedback of low beta band components: frequency-specific effects on variables of attention and event-related brain potentials. Clinical Neurophysiology, 115, 131- 139.

doi: $10.1016 /$ S1388-2457(03)00353-5

Fox, J. G. (1971). Background Music and Industrial Efficiency - A Review. Applied Ergonomics, (June), 70-73. doi: 10.1016/0003-6870(71)90072-X

Gramopadhye, A. K., \& Wilson, K. (1997). Noise, feedback training, and visual inspection performance. International J ournal of Industrial Ergonomics, 20, 223230. doi: 10.1016/S0169-8141(96)00051-0

Gluckman, J. P. (1990). Changing task demands in sustained attention: Effects on performance and perceived workload. (Doctoral Dissertation, University of Cincinnati, Cincinnati, United States of America, 1990). Retrieved from ProQuest Dissertations and Theses. (UMI No. 9108613).

Kelley, P. L. (1995). Stress Management Strategies in the Workplace; A Metaanalysis of Stress Intervention. (Doctoral Dissertation, The Pennsylvania State University, Pennsylvania, 1995). Retrieved from ProQuest Dissertations and Theses. (UMI No. 9600200).

Lehrer, P. M. (2007). Biofeedback training to increase heart rate variability. In P. M. Lehrer, R. L. Woolfolk, \& W. E. Sime, (Eds.). Principles and Practice of Stress Management ( $3^{\text {rd }}$ ed., pp. 227-248). New York: The Guilford Press. 
Lehrer, P. M., Vaschillo, E. G., Vaschillo, B., Lu, S., Eckberg, D. L., \& Edelberg, R. (2003). Heart Rate Variability Biofeedback Increases Baroreflex Gain and Peak Expiratory Flow. Psychosomatic Medicine, (65), 796-805.

doi: 10.1097/01.PSY.0000089200.81962.19

Lehrer, P. M., Vaschillo, E. G., \& Vaschillo, B. (2000). Resonant Frequency Biofeedback Training to Increase Cardiac Variability: Rationale and Manual for Training. Applied Psychophysiology and Biofeedback, 25 (3), 177-191.

doi: 10.1023/A: 1009554825745

Lehrer, P. M., Carr, R. E., \& Smetankine, A. (1997). Comparison of respiratory sinus arrhythmia and neck/trapezius EMG biofeedback for asthma: A pilot study. Applied Psychophysiology and Biofeedback, 22, 95- 109.

doi: 10.1023/A: 1026224211993

Marsh, R., Sebrechts, M. M., Hicks, J. L., \& Landau, J. D. (1997). Processing strategies and secondary memory in very rapid forgetting. Memory \& Cognition, 25, 173- 181.

McCraty, R. (2002). Influence of cardiac afferent input on heart-brain synchronization and cognitive performance. International Journal of Psychophysiology, 45 (1-2), 72-73.

McCraty, R. (2003). The scientific role of the heart in learning and performance. (Publication No. 02-030, 2003). Boulder Creek, CA: HeartMath Research Center, Institute of HeartMath. Retrieved November $10^{\text {th }}, 2007$, from

http://www. heartmath.org/templates/ihm/section_includes/education/pdf/scientifi c role_of the heart.pdf.

McCraty, R., \& Tomasino, D. (2004, November) Heart Rhythm Coherence Feedback: A New Tool for Stress Reduction, Rehabilitation, and Performance Enhancement. Paper presented at the First Baltic Forum on Neuronal Regulation and Biofeedback, Riga, Latvia. Retrieved October $30^{\text {th }}, 2007$ from http://www. heartmath.com/health/professional/hrv_biofeedback.pdf.

Megaw, E. D. (1979). Factors affecting visual inspection accuracy. Applied Ergonomics, (July), 27-32. doi: 10.1016/0003-6870(79)90006-1 
Moss, D. (2004). Heart Rate Variability Biofeedback. Psychophysiology Today - hte mind body magazine, Issue 1, 4 - 11. Retrieved November $25^{\text {th }}, 2007$ from http://www.bfe.org/articles/issuel_final.pdf.

Papadelis, C., Papadeli, K. C., Bamidis, P., \& Albani, M. (2007). Effects of imagery training on cognitive performance and use of physiological measures as an assessment tool of mental effort. Brain and Cognition, 64, 74-85.

doi: 10.1016/j.bandc. 2007.01.001

Pattyn, N., Neyt, X., Henderickx, D., \& Soetens, E. (2008). Psychophysiological investigation of vigilance decrement: Boredom or cognitive fatigue? Physiology \& Behavior, 93, 369-378. doi: 10.1016/j.physbeh.2007.09.016

Prinzel, L. J., Pope, A. T., \& Freeman, F. G. (2001). Application of Physiological SelfRegulation and Adaptive Task Allocation Techniques for Controlling Operator Hazardous States of Awareness. (Publication NASA/TM-2001-211015). Langley Research Center, Hampton, Virginia.

Rauscher, F. H., Shaw, G. L., \& Ky, K. N. (1993). Music and spatial task performance. Nature, 365, 611. doi: 10.1038/365611a0

Rowe, D. W., Sibert, J., \& Irwin, D. (1998). Heart Rate Variability: Indicator of User State as an Aid to Human-Computer Interaction. Proceeding of the SIGCHI conference on Human factors in computing systems, 480-487. Paper presented at the Conference on Human Factors in Computing Systems, Los Angeles, USA.

Schwartz, N. M., \& Schwartz, M. S. (2003). Definitions of Biofeedback and Applied Psychophysiology. In M. S. Schwartz \& F. Andrasik (Eds), Biofeedback: A practicioner's guide ( $3^{\text {rd }}$ ed., pp. 27-39). New York: The Guilford Press.

Sherman, R. A. (2004). What are Psychophysiological Assessment and Biofeedback? Retrieved December $20^{\text {th }}, 2007$ from http://www. behavmedfoundation.org/pdf/BFBSept04.pdf.

Strack, B. W. (2003). Effect of Heart Rate Variability (HRV) Biofeedback on Batting Performance in Baseball. (Doctoral Dissertation, Alliant International University, San Diego, 2003). Retrieved from ProQuest Dissertations and Theses. (UMI No. 3083450). 
Sutarto, A.P., Abdul Wahab, M.N. (2008, September). Biofeedback Technique for improving Human Operators' Cognitive Performance. Paper presented at the Fifth International Cyberspace Conference on Ergonomics, Sarawak, Malaysia.

Sutarto, A.P., Abdul Wahab, M.N., \& Mat Zin, N. (2009, October). Smart Kit for Operators' Performance. Poster session presented at the International Exposition of Research and Invention of Institutions of Higher Learning 2009, Kuala Lumpur, Malaysia.

Suvorov, N. (2006). Psychophysiological Training of Operators in Adaptive Biofeedback Cardiorhythm Control. The Spanish Journal of Psychology, 9 (2), 193-200. Retrieved July $1^{\text {st }}, 2008$ from http://www.ucm.es/info/Psi/docs/journal/v9_n2_2006/art193.pdf.

Vaschillo, E. G., Lehrer, P. M., Rishe, N., \& Konstantinov, M. (2002). Heart rate variability biofeedback as a method for assessing baroreflex function: a preliminary study of resonance in the cardiovascular system. Applied Psychophysiology and Biofeedback, 27, 1-27. doi: 10.1023/A: 1014587304314

Vaschillo, E. G., Vaschillo, B., \& Lehrer, P. M. (2006). Characteristics of resonance in heart rate variability stimulated by biofeedback. Applied Psychophysiology and Biofeedback, 31 (2), 129-142. doi: 10.1007/s10484-006-9009-3

Vernon, D. J. (2005). Can Neurofeedback Training Enhance Performance? An Evaluation of the Evidence with Implications for Future Research. Applied Psychophysiology and Biofeedback, 30 (4), 347 - 364.

doi: 10.1007/s10484-005-8421-4

Wetherell, A. (1997). Cognitive and Psychomotor Performance Tests and Experiment Design in Multiple Chemical Sensitivity. Environmental Health Perspectives, 105 (Supplement 2), 495-503. doi: 10.2307/3433359

Willis, S. L., Tennstedt, S. L., Marsiske, M., Ball, K., Elias, J., Koepke, K. M., Morris, J. N., Rebok, G. W., Unverzagt, F. W., Stoddard, A. M., \& Wright, E. (2006). Long-term Effects of Cognitive Training on Everyday Functional Outcomes in Older Adults. Journal of American Medical Association 296 (23), 2805-2814. Retrieved December $20^{\text {th }}, 2006$, from http://lang. dailynews.com/socal/dnfiles/jama.pdf. 
Article's contents are provided on a Attribution-Non Commercial 3.0 Creative commons license. Readers are allowed to copy, distribute and communicate article's contents, provided the author's and Journal of Industrial Engineering and Management's names are included. It must not be used for commercial purposes. To see the complete license contents, please visit http://creativecommons.org/licenses/by-nc/3.0/. 